\title{
A PRÁTICA EXTENSIONISTA E A ECONOMIA SOLIDÁRIA NA INCUBADORA DE TECNOLOGIAS SOCIAIS E SOLIDÁRIAS
}

\begin{abstract}
The extensionist practice and the solidary economy in the incubator of social and solidary
\end{abstract} technologies

DOI: $10.30612 /$ re-ufgd.v6i11.9265

Ijean Gomes Riedo

Alexandre Coradini Ribeiro Tania Cristina Costa Calarge

Recebido em: 15/01/2019 Aceito em: 29/11/2019

Resumo: A Incubadora de Tecnologias Sociais e Solidárias - ITESS é um setor da Pró-Reitoria de Extensão e Assuntos Comunitários voltados para o fomento e incubação de empreendimentos em Economia Solidária, pautados nos pilares de Autogestão, Gestão Científica, Incubação, Formação Cidadã, Segurança Alimentar, Agroecologia e Produção Orgânica e Questões de Gênero. Para Singer (2002) as formas alternativas de economia surgiram ainda no final do século XIX com o empobrecimento dos artesãos devido aos graves danos sociais causados pelo capitalismo industrial. Sendo que estas ações são fortemente pautadas na educação popular, cabe às Universidades fortalecer estes grupos, oferecendo qualificação técnica, em diversas áreas desde a gestão até às áreas técnicas de produção, como produção de alimentos desde a terra até o consumo (aspectos nutricionais). Diante disso os projetos desenvolvidos atualmente estão atrelados também à Agricultura Familiar. A metodologia de trabalho da Incubadora é dinâmica e se adapta a partir das demandas sociais, oportunidades de financiamento e parcerias com organizações dos diversos setores - sempre através de projetos de extensão, sendo que em todas as perspectivas de alternativa social e econômica se mantém. Muitos são os desafios da prática extensionista, mas podem-se destacar três principais no modelo da ITESS: 1) a infraestrutura ofertada para que os grupos sociais iniciem sua atividade produtiva é adaptada de um espaço de ensino, e como tal, possui limitações físicas, bem como limitações de investimento e financiamento; 2) Pela característica de incubação através de projetos, muitas demandas dos grupos sociais e da comunidade acadêmica envolvida surgem depois do início das atividades, o que provoca a necessidade de ajustes no projeto inicial, ou a submissão de novos projetos para atender novas demandas, antes não previstas. E por fim, 3) ainda são pouco conhecidas as práticas da ITESS/UFGD pela comunidade interna e externa, apesar de alguns projetos incubados se destacarem. Destaca-se este último desafio, pois o aumento da procura pelos serviços da incubadora, inclusive como oportunidade de extensão pelo público universitário, possibilitaria o efetivo compromisso da extensão universitária, colocando os acadêmicos em contato com a comunidade e colocando em prática e desenvolvendo seus conhecimentos. Observa-se ainda que as cooperativas - que foram o primeiro tipo de empreendimento a romper os paradigmas de Capital e Trabalho - ainda não são um público atendidos pela ITESS/UFGD sendo, portanto, uma oportunidade para projetos futuros.

Palavras-chave: Economia Solidária; Geração de Renda; Incubadora Social. 
Abstract: The Social and Solidarity Technologies Incubator - ITESS is a sector of the Pro-Rectory of Extension and Community Affairs focused on the development and incubation of enterprises of Solidarity Economy, based on the pillars of Self-management, Scientific Management, Incubation, Citizen Training, Security Food, Agroecology and Organic Production and Gender Issues. For Singer (2002) the alternative forms of economy still appeared in the late nineteenth century with the impoverishment of artisans due to the serious social damages caused by industrial capitalism. Since these actions are strongly based on popular education, it is up to the universities to strengthen these groups, offering technical qualification in various areas from management to technical areas of production, such as food production from land to consumption (nutritional aspects). In view of this, the projects currently under development are also linked to Family Agriculture. The Incubator's work methodology is dynamic and adapts based on social demands, financing opportunities and partnerships with organizations from the various sectors - always through extension projects, and in all perspectives of social and economic alternatives is maintained. Many are the challenges of the extensionist practice, but three main ones can be highlighted in the ITESS model: 1) the infrastructure offered for the social groups to start their productive activity is adapted from a teaching space, and as such has physical limitations, as well as investment and financing constraints; 2) Due to the characteristic of incubation through projects, many demands of the social groups and the academic community involved arise after the beginning of the activities, which causes the need for adjustments in the initial project, or the submission of new projects to meet new demands, before not foreseen. And lastly, 3) the ITESS/UFGD practices by the internal and external community are still little known, although some incubated projects stand out. This last challenge stands out because increasing the demand for incubator services, including as an opportunity for extension by the university public, would make possible the effective commitment of university extension, placing the students in contact with the community and putting into practice and developing their knowledge. It is also observed that cooperatives - which were the first type of enterprise to break the paradigms of Capital and Work - are not yet an served by ITESS/UFGD and, therefore, an opportunity for future projects.

Keywords: Solidarity economy; Income Generation; Social Incubator.

\section{Introdução}

A Economia Solidária advoga e trabalha por uma base mais justa, solidária, coletiva da produção e distribuição de benefícios, dispensando atenção primordial aos interesses de melhoria de qualidade de vida dos trabalhadores por eles mesmos. Os empreendimentos solidários consideram o coletivo e incentivam a colaboração ao invés da competição (ANTEAG, 2005). O desenvolvimento da Economia Solidária e seus princípios na Universidade envolve um processo lento de educação, formação, capacitação e qualificação permanente e integral, com seu tratamento especialmente em incubadoras sociais.

As Incubadoras Sociais não só tem um impacto na sociedade, mas atuam de forma contínua, e transborda para a sociedade o conhecimento que é gerado nos âmbitos acadêmico, 
governamental e empresarial. Entendemos que o desenvolvimento tecnológico e econômico não está dissociado ao desenvolvimento social e do cuidado ambiental. Os impactos na sociedade que podem ser engendrados por este tipo de incubadora são diversos, podendo ser refletidos nos espaços social, econômico e cultural (BEZERRA et al., 2014).

Partindo da premissa que para a redução da desigualdade social vivenciada pelas comunidades locais, formas de inovação e inserção precisam ser dadas em direção a um desenvolvimento sustentável, no quais processos mercadológicos injustos, desiguais, opressores e alienadores são transformados em Economia Solidária, sintetizadas em justiça, humanidade, criatividade, cooperação, alinhamento e respeito aos ciclos da natureza provedora (SIMONI, 2010).

No entanto, essas ações apresentam duas características comuns à economia solidária e à sua estruturação acadêmica no Brasil (CALBINO e PAULA, 2013). A primeira delas é a marcante primazia de sua ocorrência nas universidades como projetos de extensão, e nesses, como projetos de extensão de conhecimentos técnicos, sendo a economia solidária um elemento acessório nesses projetos, e não seu elemento de reflexão central. O segundo é a descontinuidade, tanto na produção acadêmica quanto nas políticas públicas voltadas para a economia solidária no município.

Tal fato pode ser explicado pela submissão, tanto das políticas públicas, quanto das ações acadêmicas voltadas para a economia solidária, às concepções e prioridades das gestões tanto dos entes estatais, como a Prefeitura de Dourados, quanto das gestões das Universidades, visto que as incubadoras voltadas ao desenvolvimento de projetos de economia solidária nessas instituições se estruturaram como setores das Pró-Reitoria de extensão como é comum acontecer nas universidades brasileiras (FARIAS, 2013).

Nesse sentido, embora se estruturem de forma a buscar a articulação entre ensino, pesquisa e extensão, de forma a buscar uma formação crítica de profissionais e pesquisadores, e uma intervenção transformadora na sociedade (CULTI, 2007), essa busca pode encontrar dificuldades, principalmente em função da confusão que se estabelece entre os princípios da economia solidária, e sua aplicação no processo de incubação, como os princípios que vão reger outras incubadoras, como as incubadoras tecnológicas e as incubadoras de empreendimentos mercantis tradicionais (RIOS, 2017).

Esse estudo questiona o desenvolvimento do papel extensionista para a promoção da Economia Solidária através das Incubadoras de Tecnologias Sociais e Cooperativas Populares de Universidades de Dourados/MS (DUBEUX, 2007). Para isso foi realizado ensaio (FARIA, 2017) no âmbito dos princípios da Economia Solidária a lógica da sustentabilidade econômica por meio de 
processos de trabalho estruturados em função de elementos de autogestão e solidariedade.

\section{Referencial Teórico}

A Economia Solidária se caracteriza por concepções e práticas fundadas em relações de colaboração solidária, inspiradas por valores culturais que colocam o ser humano na sua integralidade ética e lúdica e como sujeito e finalidade da atividade econômica, ambientalmente sustentável e socialmente justa, ao invés da acumulação privada de capital. Esta prática de produção, comercialização, finanças e de consumo, privilegia a autogestão, a cooperação, o desenvolvimento comunitário e humano, a satisfação das necessidades humanas, a justiça social, a igualdade de gênero, raça, etnia, acesso igualitário à informação, ao conhecimento e a segurança alimentar, preservação dos recursos naturais pelo manejo sustentável e responsabilidade com as gerações, presente e futura, construindo uma nova forma de inclusão social com a participação de todos (SENAES, 2015).

É importante ressaltar que também as noções de cooperativismo e associativismo, presentes em estudos em instituições com forte desenvolvimento da extensão rural, embora próximas, não expressam todas as possibilidades presentes na economia solidária, gerando uma série de mal entendidos (SINGER, 2012). Dentre elas, um elemento que tem se mostrado altamente relevante atualmente é a questão da informalidade, que deixa de ser percebida como necessariamente um problema, ou um impedimento para a expansão da economia solidária, ou de seus empreendimentos (SENAES, 2012).

\section{Conceitos de Economia Solidária}

As discussões sobre economia solidária pode ser fragmentada em três distintas proposições: (i) a economia solidária é um novo modo de produção paralelo ao modo capitalista de produção; (ii) o cooperativismo é um movimento que se desenvolve no interior do capitalismo, integrando-se a ele com uma forma diferenciada de organização; (iii) As cooperativas de trabalhadores são rupturas no interior da forma de produção capitalista, mas não constituem uma nova forma de produção, senão uma contradição da forma de organização tipicamente capitalista de trabalho e produção (SINGER, 2002; 2012).

A forma histórica de organização cooperativista é tanto uma reação defensiva dos trabalhadores diante das condições adversas econômicas e sociais impostas pelo capitalismo e pelo socialismo utópico. Nesse sentido, os EES fazem oposição e resistência ao modelo capitalista de 
produção, enquanto estratégia defensiva que se desenvolve paralelamente ao modo dominante, quanto as diferentes formas de inserção alternativa no sistema de capital (FARIA, 2017).

No Brasil foi criado em 2003 a Secretaria Nacional de Economia Solidária (SENAES), institucionalizando a expressão “economia solidária”. A proposição, pelo governo federal, era de uma política enquanto mecanismo de consolidação de organizações populares de geração de emprego e renda por meio de grupos comunitários, associações ou cooperativas numa mesma posição de formas diferenciadas de unidades produtivas (SENAES, 2004).

Convém observar que a finalidade da Senaes é promover o fortalecimento e a divulgação da economia solidária mediante políticas integradas, visando ao desenvolvimento por meio da geração de trabalho e renda com inclusão social (SENAES, 2012).

Tendo seus princípios baseado na busca por um modelo de organização que vise sustentar relações igualitárias, através na participação democrática da gestão, onde o processo de tomada de decisão é realizado de forma transparente e de forma compartilhada (CATALISA, 2005). Este modelo corresponde a uma organização coletiva na qual o elemento essencial é a democracia, envolvendo a participação integral dos membros do grupo, acesso total às informações, conhecimento dos processos e, sobretudo, autonomia e autodeterminação (ANTEAG, 2009).

A autogestão com base na economia solidária, lida com a realidade vivida pelos trabalhadores excluídos do mercado formal de trabalho, que constituem novos empreendimentos coletivos de produção e serviços, ou que assumem empresas falidas ou em dificuldades (SINGER, 2002).

Vale lembrar ainda que a nova política econômica moderna passou a valorizar os empreendimentos locais como forma de inserção no trabalho formal e também na sociedade, daqueles que foram excluídos pelo novo modelo de produção capitalista, fazendo recorrer na análise da economia solidária (DAGNINO, 2014).

\section{Tecnologias Sociais}

Entende-se por “tecnologias sociais”, “[...] o conjunto de técnicas e metodologias transformadoras, desenvolvidas e/ou aplicadas na interação com a população e apropriadas por ela, que representam soluções para inclusão social e melhoria das condições de vida" (INSTITUTO DE TECNOLOGIA SOCIAL, 2007, p. 29). O vocábulo, grafado no plural, representa aqui o entendimento de que existem múltiplas formas de tecnologia social, assim como múltiplos são os enclaves sociais e as identidades culturais. 
As tecnologias sociais, para Dagnino (2004), caracterizam-se pelo(a): (i) adaptação a pequeno tamanho físico e financeiro; (ii) caráter inclusivo; (iii) orientação para o mercado interno de massa; (iv) libertação do potencial e da criatividade; e (v) capacidade de viabilizar economicamente os empreendimentos autogestionários e a economia solidária. Produz, ademais, forte interação teoria-prática, visto que parte de problemas diretamente relacionados ao ambiente local de uma comunidade e permite a construção de conhecimento coletivo e passível de apropriação crítica.

\section{Materiais e Métodos}

Nesta pesquisa foi realizado ensaio teórico. O ensaio precisa ser utilizado como opção consciente e intencional, ou seja, como a forma mais adequada no entendimento de algo. O que se verifica, na atualidade, é que o ensaio vem atender a um apelo da sociedade do consumo cultural e midiática: Vale tudo para se expressar (MENEGHETTI, 2011).

A utilização do ensaio como forma não significa a total rendição ao fim dos limites formais ou a crítica irracional que se possa fazer em relação à ciência, mas uma forma específica de compreensão da realidade, por meios diferentes daqueles utilizados pela ciência, na sua forma tradicional de produzir conhecimento. Assim, o ensaio caracteriza-se pela sua natureza reflexiva e interpretativa, diferente da forma classificatória da ciência (FARIA, 2017).

No centro do ensaio está a relação quantitativa versus qualitativa. Enquanto a ciência adquire maior autonomia, valorizando aspectos quantitativos para promover generalizações que façam com que um número cada vez maior de pessoas passe a compreender o mundo a partir da instituição de uma racionalidade baseada na calculabilidade, o ensaio valoriza aspectos relacionados às mudanças qualitativas que ocorrem nos objetos ou fenômenos analisados pelos ensaístas (MENEGHETTI, 2011).

\section{Resultados e Discussão}

A cidade de Dourados se encontra como um cenário de grande desenvolvimento desse tipo de ações, possuindo além de diversos grupos que atuam sob o modelo da economia solidária, também um banco comunitário (Pirê) com moeda social, um Secretaria Municipal de Economia Solidária (hoje anexada à Secretaria de Agricultura), e entidades articulação, como o Fórum e o Conselho Municipal de Economia Solidária. Da mesma forma, as instituições Públicas de Ensino Superior da Cidade, possuem incubadoras voltadas para o fomento de iniciativas na área 
de economia solidária, como a Incubadora de Tecnologias Sociais e Solidárias na UFGD; a ELOS, na UEMS, e a Incuba Social da Grande Dourados, ligada ao IFMS de Dourados. Além disso, podem-se perceber diversos projetos acadêmicos nessas instituições voltados para mobilização de grupos sociais que se utilizam, ainda que indiretamente, elementos da economia solidária nas suas formulações teóricas e metodológicas.

\section{Papel extensionista: Economia Solidária em Dourados}

A ONG Mulheres em Movimento, em parceria com a Secretaria de Assistência Social e Economia Solidária do Município de Dourados e as Universidades locais (através das Incubadoras Sociais), impulsionou a criação da Rede de Economia Solidária de Dourados. Foram realizados levantamentos sobre o consumo e produção da população, incluindo os insumos utilizados na produção, os locais onde produtores e consumidores estão realizando suas compras e a localização das indústrias, comércio e serviços (COSTA, 2011).

O objetivo da rede de economia solidária, além de gerar trabalho, renda e inclusão social, é de orientar a linha de crédito do banco Pirê, banco criado pela ONG Mulheres em Movimento para apoiar a economia solidária na região de Dourados, priorizar o financiamento de bens e serviços, evitar a competição entre os integrantes da rede e reduzir os custos de produção a partir de compras conjuntas. As ações se apoiam num mapa de produção que possibilita a montagem das cadeias produtivas onde cada empreendedor produz em função das necessidades de insumos do outro.

Há uma preocupação quanto à qualidade e padronização dos produtos e serviços prestados pela Rede. Tal preocupação torna necessário o investimento na qualificação, requalificação e capacitação dos empreendedores (PAULON, 2010).

A Rede de Economia Solidária é uma organização civil e democrática de trabalhadores e consumidores solidários, com a finalidade de gerar trabalho e renda, inclusão social e produtiva a partir dos princípios da Economia Solidária. Ela está estruturada por região dentro do município. Um conjunto de bairros próximos forma uma região, onde os integrantes se reúnem todos os meses para articular atividades, discutir e aprofundar temas de interesse (SEGUNDO, 2014).

Informações da Secretaria de Assistência Social e Economia Solidária de Dourados indicam que mais de oito mil trabalhadores foram qualificados em diversos cursos, entre 2001 a 2008, por meio do Programa Coletivo de Qualificação para o Trabalho, que fez um investimento de 
mais de dois milhões de reais.

\section{Órgãos de Apoio}

Segundo o Ministério do Trabalho e Emprego consideram-se entidades de apoio, assessoria e fomento para a Economia Solidária as organizações públicas e privadas sem fins lucrativos que desenvolvem ações nas várias modalidades de apoio direto, capacitação, assessoria, incubação, assistência técnica e de gestão e acompanhamento junto aos empreendimentos de Economia Solidária.

A ONG Mulheres em Movimento é uma entidade sem fins lucrativos, fundada em janeiro de 2004, que tem por finalidade apoiar a Rede de Economia Solidária de Dourados, através de acompanhamento técnico e suporte financeiro aos empreendimentos solidários, bem como trabalhar com a formação cidadã dos empreendedores.

Os recursos da ONG são provenientes da doação de bens e da herança de uma jovem missionária e de outros doadores voluntários. Em Julho de 2006, a ONG Mulheres em Movimento criou o Banco Comunitário de Desenvolvimento, denominado Banco Pirê, integrando a Rede Brasileira de Bancos Comunitários. O nome do Banco e de seus produtos tem origem na etnia Tupi Guarani, povo indígena nato do Município de Dourados. O Banco Pirê lançou a moeda social denominada Pirapirê, palavra que simboliza o "dinheiro" na etnia guarani e quer dizer abundância. “ Pira significa peixe e Pirê casca ou escama de peixe" (BANCO PIRÊ, 2006).

O Banco Pirê é um sistema integrado que organiza os moradores de Dourados para se articularem em rede, produzirem e consumirem na própria cidade. Muito além de concessão de microcrédito, o Banco Pirê é um programa de desenvolvimento local, tendo como início a capacitação e empoderamento dos empreendedores, despertando o senso para a solidariedade e a colaboração como um modelo de desenvolvimento justo e sustentável. Além de usar apenas instrumentos de crédito, integra consumo, produção e comercialização, que permite a ligação entre as cadeias produtivas locais e a geração de trabalho e renda aos moradores.

Através de reuniões nos bairros, o Banco Pirê divulga suas ações e mobiliza os moradores para inserir-se a rede de Economia Solidária. O processo de construção da rede teve início a partir do mapeamento de produção e do consumo da região. O banco disponibiliza um sistema de microcrédito para estimular a produção local e satisfazer a demanda do consumo existente através de cartão de crédito, feiras, lojas para que os consumidores de um bairro comprem os produtos produzidos no local. A moeda disponibilizada neste sistema pode ser em moeda social 
ou moeda oficial, dependendo do caso.

A concessão de crédito é feita através da análise pelo(a) monitor(a) do bairro que faz o levantamento sobre o empreendedor e dá seu aval através de uma declaração escrita para o Banco Pirê. A partir daí o Banco entra em contato com esse empreendedor por meio de visita, entrevista e levantamento sobre sua participação junto à rede e o perfil profissional.

\section{Secretaria Municipal de Assistência Social e Economia Solidária}

Economia Solidária foi política prioritária desde 2001 em Dourados para inclusão social, geração de trabalho e renda, e desenvolvimento local. As atividades realizadas pela prefeitura foram a implementação de assessoria técnica para legalização de quase trezentos empreendimentos, realização de cursos do Programa Coletivos de Qualificação para o Trabalho, atendendo mais de 8.000 pessoas, elaboração do pré-projeto para a criação de lei municipal de fomento, a ECOSOL, capacitação continuada em economia solidária, tanto para a equipe técnica como para a Rede, apoio as feiras e lojas solidárias e finalmente o fortalecimento da Rede de Economia Solidária.

A Equipe Técnica contou com profissionais de diversas áreas, como ambientalistas, contadores, modistas, estilistas, engenheiros, programadores, etc., para melhorar a qualidade da produção, dos produtos, do ambiente de trabalho e da comercialização. Um exemplo é a padronização dos tamanhos e modelos das peças de roupa produzidas.

\section{Universidade Estadual do Mato Grosso do Sul - UEMS}

A UEMS foi escolhida em 2007 para realizar o mapeamento dos empreendimentos econômicos e solidários no Mato Grosso do Sul, dando continuidade a um trabalho iniciado em 2005, pela Universidade Federal de Mato Grosso do Sul - UFMS. O objetivo era construir um Sistema Nacional de Informações em Economia Solidária (SIES) visando fortalecer os processos de formulação de políticas publicas para o desenvolvimento da Economia Solidária. Segundo o catálogo do mapeamento (2007) foi entrevistados na região da Grande Dourados 132 empreendimentos, sendo 120 somente no município de Dourados-MS, Além desse levantamento, a UEMS abriga a incubadora ELOS/ITCP - Incubadora de Tecnologia Social para Cooperativas Populares que tem a missão de combater a pobreza com a inclusão econômica. A incubadora tem o intuito de apoiar grupos que queiram se organizar através a formação de Empreendimentos Econômicos solidários e sustentáveis, como cooperativas, Associações e outros grupos. 


\section{Universidade Federal da Grande Dourados - UFGD}

A UFGD participou da proposta de um projeto denominado como Implantação Tecnológica de Cooperativas Populares - ITCP - na região da grande Dourados, região sul de Mato Grosso do Sul, que teve como objetivo implantar uma Incubadora Tecnológica de Cooperativas Populares envolvendo as áreas de ensino, pesquisa e extensão. Os Empreendimentos Econômicos Solidários objeto da proposta da UFGD são:

- Grupo de Mulheres Quilombolas;

- Grupo de mulheres do Assentamento Lagoa Grande.

Após o protagonismo de docentes da UFGD para a implantação foi constituída e fundada como um programa de extensão universitária e incorporada à estrutura administrativa da UFGD, em 2006, a Incubadora de Tecnologias Sociais e Solidárias ITESS/UFGD, que desenvolve ações com Empreendimentos de Economia Solidária (EES) nos âmbitos sociais, econômicos e políticos, pautadas em um processo educativo dialógico estruturado nos princípios da economia solidária.

A ITESS atua por meio de um núcleo interdisciplinar e multidisciplinar organizado pela atuação de um quadro formado por docentes, acadêmicos/as, técnicos/as, movimentos sociais e outras instituições, buscando assim, socializar o conhecimento e ampliá-lo para rumos mais solidários objetivando a transformação da sociedade e da própria universidade. Desse modo, a Incubadora prima pela geração de trabalho e renda, com princípios de formação política, ampliação e troca de saberes, enfim, desenvolve reflexões acerca da conquista de cidadania e direitos humanos.

\section{Fatores limitantes e potencialidades}

A metodologia de trabalho da Incubadora é dinâmica e se adapta a partir das demandas sociais, oportunidades de financiamento e parcerias com organizações dos diversos setores - sempre através de projetos de extensão, sendo que em todas as perspectivas de alternativa social e econômica se mantém.

Muitos são os desafios da prática extensionista, mas podem-se destacar três principais no modelo da ITESS:

Quadro 1 - Pontos fortes e fracos do desenvolvimento de práticas em Incubadoras Sociais 
Infraestrutura

Metodologia de incubação

Público-alvo
A infraestrutura ofertada para que os grupos sociais iniciem sua atividade produtiva é adaptada de um espaço de ensino, e como tal, possui limitações físicas, bem como limitações de investimento e financiamento.

Pela característica de incubação através de projetos, muitas demandas dos grupos sociais e da comunidade acadêmica envolvida surgem depois do início das atividades, o que provoca a necessidade de ajustes no projeto inicial, ou a submissão de novos projetos para atender novas demandas, antes não previstas.

Ainda são pouco conhecidas as práticas da ITESS/UFGD pela comunidade interna e externa, apesar de alguns projetos incubados se destacarem.

Fonte: Elaborado pelos autores, a partir Alves et al. (2016).

Destaca-se este último desafio, pois o aumento da procura pelos serviços da incubadora, inclusive como oportunidade de extensão pelo público universitário, possibilitaria o efetivo compromisso da extensão universitária, colocando os acadêmicos em contato com a comunidade e colocando em prática e desenvolvendo seus conhecimentos.

\section{Considerações Finais}

Os grupos autogestionárias são organizações baseadas no trabalho coletivo, configurando-se como uma organização, na qual ocorre à associação de pequenos produtores no desenvolvimento das atividades de produção para se estabelecer no mercado local, onde partes dos ganhos são repartidos entre os membros e a outra convertidos em benefícios para a região onde se encontram estabelecida, não levando em conta sua estrutura interna de gestão, mas sim a qualidade e a eficiência dos produtos e serviços oferecidos, que garantiram sua sobrevivência e sua autosustentação no mercado.

Outro ponto que merece destaque é participação efetiva da mulher neste arranjo produtivo, pois o que se observa hoje, é que o trabalho das mulheres em geral, é percebido como 
ajuda ao marido ou ao pai. Muitas vezes não é reconhecido como trabalho que gera renda para a família. Quando as mulheres são remuneradas, recebem um rendimento menor se comparado ao dos homens. Por outro lado, as mulheres dedicam a maior parte do seu tempo nas atividades voltadas para o autoconsumo, que não são reconhecidas como atividades geradoras de renda. Por fim, a obtenção de renda pelas mulheres é influenciada pelo baixo grau de agregação de valor aos produtos que elas comercializam. Quando organizadas em grupos produtivos, estes carecem de infraestrutura produtiva, de qualificação para o gerenciamento, organização dificultando o acesso aos mercados consumidores (COSTA, 2011).

No âmbito do espaço doméstico, o trabalho produtivo desenvolvido pelas mulheres, normalmente não remunerado fica escondido, invisível por detrás das estratégias de produção familiar (LOMBARDI, 2009). Esse trabalho invisível, no entanto, representa uma grande contribuição das mulheres na produção para o consumo próprio e da família. Por outro lado, quando as mulheres participam dos diferentes espaços de produção seu trabalho é visto como ajuda, complementar ao trabalho dos homens. A invisibilidade do trabalho doméstico das mulheres tornou-se então, o foco do debate da economia feminista.

Uma forma de fortalecer e ampliar a participação da mulher na economia é a organização de grupos, associações e cooperativas - visando fortalecer e ampliar processos produtivos e de comercialização. Experiências indicam que produtores organizados, em suas mais diferentes formas de ação coletiva, obtêm melhores oportunidades de participarem de mercados de forma mais eficiente. Essas formas de ações coletivas podem reduzir os custos de transações de acesso aos insumos e aumentar o poder de negociação.

Observa-se ainda que as cooperativas - que foram o primeiro tipo de empreendimento a romper os paradigmas de Capital e Trabalho - ainda não são um público atendidos pela ITESS/UFGD sendo, portanto, uma oportunidade para projetos futuros.

\section{Referências}

ALVES, J. N.; FLAVIANO, V.; KLEIN, L. L.; LÖBLER, M. L.; PEREIRA, B. A. D. (2016). A economia solidária no centro das discussões: um trabalho bibliométrico de estudos brasileiros. Cad. EBAPE.BR vol.14 no.2 Rio de Janeiro-RJ. http://dx.doi.org/10.1590/1679-395120257.

ANTEAG (2005). Autogestão e economia solidária: uma nova metodologia. Vol. 2, Brasília: TEM, 2005.

BEZERRA, A. F. A.; SILVA, W. S. C.; CARVALHO, Z. V. (2014). As Incubadoras Sociais e o 
porque

apoiar

a iniciativa. anprotec.org.br/anprotec2014/files/artigos/artigo\%20(57).pdf

CALBINO, D; PAULA, A. P. P. (2013). Economia Solidária: investigação sobre o estado da arte. Gestão Contemporânea, 10(14), 371-397. Consultado em 20 de março de 2017 de http://seer4.fapa.com.br/index.php/arquivo/article/viewFile/302/145.

CATALISA (2010). Rede de Cooperação para Sustentabilidade. Disponível em: $<$ http://www.catalisa.org.br>. Acesso em 28 de Maio de 2016.

COSTA, J. C. (2011) Mulheres e Economia Solidária: Hora de discutir a relação! Sociedade e Cultura (UFG), 14(1), 19-27. Recuperado em 20 de março de 2017 de https://www.revistas.ufg.br/fchf/article/view/15649/9881.

CULTI, M. N. (2007). Economia solidária: Incubadoras universitárias e processos educativos. Proposta, v.31, n. 111, p. 16- 22, 2007. Disponível em http://www.unitrabalho.uem.br/administracao/bd_artigos/arquivos/010614153016.pdf

DAGNINO, R. (2004) A tecnologia social e seus desafios. In: Tecnologia social uma estratégia para o desenvolvimento. Fundação Banco do Brasil, Rio de Janeiro-RJ.

. (2014). Tecnologia Social: Contribuições conceituais e metodológicas. Campina Grande: EDUEPB. Pag. 24-30. ISBN 978-85-7879-327-2.

DUBEUX, A. (2007). O papel das universidades na construção da economia solidária no Brasil. Proposta, v.31, n. 111, p. 3-15. Disponível em http://fase.org.br/wp-content/uploads/2007/03/proposta-111-final.pdf.

FARIA, J. H. (2017). Autogestão, economia solidária e organização coletivista de produção associada: em direção ao rigor conceitual. Cad. EBAPE.BR vol.15 no.3 Rio de Janeiro-RJ. http://dx.doi.org/10.1590/1679-395157778.

FARIAS, M. F. L. (2013). Processos educativos e ressignificação de experiências em incubação na Universidade Federal da Grande Dourados (UFGD). pags. 17-39. In: FARIAS, M. F. L; OLIVEIRA, E. R; FAISTING, A. L. (orgs). Experiências interdisciplinares para a construção de conhecimentos solidários. Dourados: Ed. UFGD. ISBN 978-85-8147-011-5.

INSTITUTO DE TECNOLOGIA SOCIAL (2007). Tecnologia Social. São Paulo: ITS/Secis-MCT, série "Conhecimento e Cidadania", no 1 .

MENEGHETTI, F. K. (2011). O que é um ensaio-teórico?. Rev. adm. contemp. vol.15 no.2 Curitiba-PR. http://dx.doi.org/10.1590/S1415-65552011000200010.

PAULON, G. B. F. S. (2010). Tecendo a igualdade: reflexões sobre gênero e trabalho na rede de economia solidária em Dourados. Dissertação de Mestrado, Programa de Pós Graduação em História da UFGD. (229f.). Consultado em 20 de marco de 2017 de http://tede.ufgd.edu.br/jspui/bitstream/tede/225/1/GrazihelyBereniceFernandesSPaulon.pdf. 


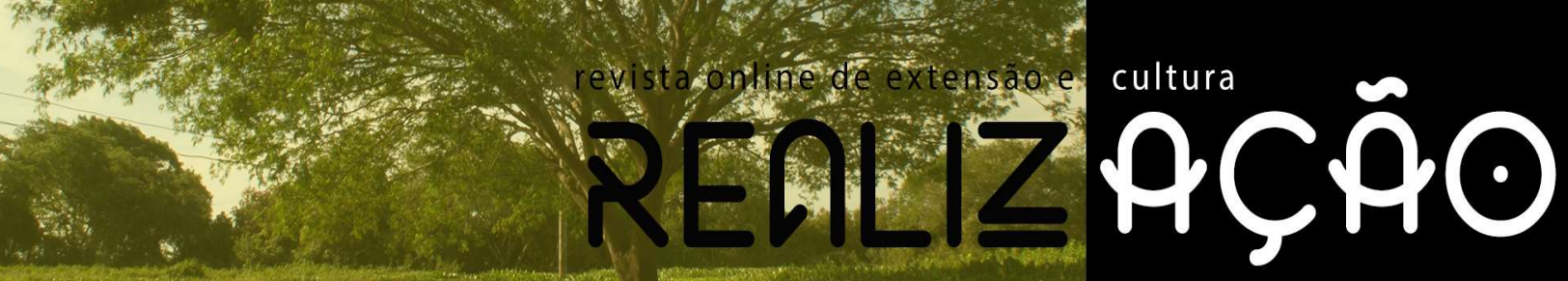

RIOS, D. M. S.; LIMA, J. R. O. (2017) A prática da extensão universitária como incentivadora da tecnologia social. Disponível em: < http://www.unitrabalho.org.br/artigos/pratica-extensaouniversitaria-como-incentivadora-tecnologia-social/>. Consultado dia 01 de agosto de 2017.

SEGUNDO, J. J. M. N. (2014). Como esta organizada a rede de Economia Solidária em Dourados. Dados da Superintendência de Trabalho e Economia Solidária - SupTES (2005). Disponível em < http://www.dourados.ms.gov.br/wp-content/uploads/2014/09/arquivo-6.pdf $>$. Consultado em 07 de outubro de 2017.

SENAES-Secretaria Nacional de Economia Solidária (2004). Manual de Entrevista. Brasília: MTE/ Senaes.

MTE/Senaes. Pag. 153.

(2012). Avanços e Desafios para as Políticas Públicas de Economia Solidária. Brasília: (2015). $1^{\circ}$ Plano Nacional de Economia Solidária. Brasília: MTE/Senaes Disponível em: http://trabalho.gov.br/images/Documentos/EconomiaSolidaria/PlanoNacionalEcoSol.pdf.

SIMONI, J. A. (2010) Revitalização do Extrativismo: Práticas de Economia Solidária e Sustentabilidade. Mercado de trabalho, v. 42.

SINGER, P. (2002). Introdução à economia solidária. Fundação Perseu Abramo.

(2012). Os oito primeiros anos da Secretaria Nacional da Economia Solidária. In: LIANZA, S.; HENRIQUES, F. C. (Orgs). A Economia Solidária na América Latina: Realidades Nacionais e Políticas Públicas. Rio de Janeiro: Pró Reitoria de Extensão UFRJ. Pag. 63-70.

VIEITEZ, C. G.; DAL RI, N. M. (2004). Elementos da história da ANTEAG. ORG \& DEMO, v.5,11.2, p.267-272. 